\title{
Modeling CHANDRA low energy transmission grating spectrometer observations of classical novae with PHOENIX
}

\section{V4743 Sagittarii}

\author{
A. Petz ${ }^{1}$, P. H. Hauschildt ${ }^{1}$, J.-U. Ness ${ }^{1}$, and S. Starrfield ${ }^{2}$ \\ 1 Hamburger Sternwarte, Gojenbergsweg 112, 21029 Hamburg, Germany \\ e-mail: [apetz; yeti; jness]@hs.uni-hamburg.de \\ 2 Department of Physics and Astronomy, Arizona State University, Tempe, AZ 58287-1504, USA \\ e-mail: starrfield@asu.edu
}

Received 3 June 2004 / Accepted 1 October 2004

\begin{abstract}
We use the PHOENIX code package to model the X-ray spectrum of Nova V4743 Sagittarii observed with the LETGS onboard the Chandra satellite 19 March 2003. Our atmosphere models are 1D spherical, expanding, line blanketed, and in full non-local thermodynamic equilibrium. To analyze nova atmospheres and related systems with an underlying nuclear burning envelope at X-ray wavelengths, it was necessary to update the code with new microphysics, as discussed in this paper. We demonstrate that the X-ray emission is dominated by thermal bremsstrahlung and that the hard X-rays are dominated by Fe and $\mathrm{N}$ absorption. The best fit to the observation is provided at a temperature of $T_{\text {eff }}=5.8 \times 10^{5} \mathrm{~K}$, with $L_{\text {bol }}=50000 L_{\odot}$. The models are calculated for solar abundances. It is shown that the models can be used to determine abundances in the nova ejecta.
\end{abstract}

Key words. stars: novae, cataclysmic variables - X-rays: individuals: V4743 Sgr - stars: atmospheres - atomic data

\section{Introduction}

Classical novae $(\mathrm{CNe})$ are the third most violent of the stellar explosions that can occur in a galaxy (after $\gamma$-ray bursts and supernovae). About 5 to 10 Galactic $\mathrm{CNe}$ are discovered per year in the Solar neighborhood. CNe participate in the cycle of Galactic chemical evolution, in which they eject grains and metal-enriched gas as a source of heavy elements for the interstellar medium (supplementing those of supernovae, AGB stars, PNe, and WR stars: Gehrz et al. 1998). In addition, $\mathrm{CNe}$ are related to the Super Soft Binary X-ray Sources (SSS) which are probably progenitors of SN Ia explosions (Kahabka \& van den Heuvel 1997) and which are thought to be the result of thermonuclear explosions in the cores of mass-accreting white dwarfs in close binary systems (Hillebrandt \& Niemeyer 2000; Leibundgut 2001). The association of CNe and SSS is important both because of the cosmological implications of SN Ia (currently the best standard candles for determining cosmological distances) and because SN Ia are thought to be responsible for the abundance of iron in the solar system.

$\mathrm{CN}$ explosions occur in a cataclysmic variable (CV) binary star system in which a Roche lobe filling secondary supplies hydrogen-rich material that is accreted onto a white dwarf (WD) primary. Theoretical studies show that the accreted layer grows until it is sufficiently hot and dense to initiate a thermonuclear runaway (TNR). The evolution of the TNR depends upon the mass and luminosity of the $\mathrm{WD}$, the rate of mass accretion, the composition of the accreted material, and the chemical composition in the reacting layers (Starrfield 1989; Gehrz et al. 1998). Observations imply and theory demands that core material be dredged-up into the accreted material and the gases be thoroughly mixed before being ejected into space (Starrfield et al. 1998; Gehrz et al. 1998). Thus, the chemical composition of the ejected material reflects a TNR processed mixture of WD core plus accreted material. Abundance studies of the ejecta carried out with the IUE satellite have established that the underlying WDs are either carbon-oxygen (CO) or oxygen, neon, and magnesium (ONeMg) WDs (Starrfield et al. 1998; Gehrz et al. 1998). CNe are expected to be the major source of ${ }^{15} \mathrm{~N}$ and ${ }^{17} \mathrm{O}$ in the Galaxy and to contribute to the abundances of other isotopes in this atomic mass range.

Previous X-ray studies of $\mathrm{CNe}$ in outburst show that no other wavelength region provides unambiguous information on the evolution and characteristics of the underlying WD (Drake et al. 2003; Krautter et al. 1996, 2002; Ness et al. 2003). For example, the ROSAT studies discovered a "soft" component in $\mathrm{CNe}$ and placed them at, or near, the bright end of the class of SSS. The "soft" component has been identified as emission from the hot nuclear burning photosphere of the WD and theoretical studies show that a determination of the temperature and lifetime is a measure of the mass of the WD. Stellar atmosphere analyses of the photosphere can provide abundances of the 
material remaining on the WD. $\mathrm{CNe}$ at optical maximum are luminous, $L \geq 10^{5} L_{\odot}$ and evolve to X-ray maximum where $T_{\text {eff }}$ ranges from $3 \times 10^{5} \mathrm{~K}$ to $6 \times 10^{5} \mathrm{~K}$ or higher (Krautter et al. 1996; Balman et al. 1998; Orio et al. 2002, 2003).

$\mathrm{X}$-ray emission during the $\mathrm{CN}$ outburst can be divided into three phases (Krautter 2002). The first is the early fireball phase, in which the hot atmosphere of the white dwarf is expanding and cooling adiabatically. The peak temperature of the white dwarf is predicted to exceed $10^{6} \mathrm{~K}$ (depending on the mass of the white dwarf: Starrfield et al. 1996) so, if detected in this phase, it would be an extremely hot source. Nevertheless, the expanding shell cools rapidly and becomes more opaque in $\mathrm{X}$-rays in a few hours. Therefore, for the first few hours of the outburst, after the shell has cooled sufficiently, the nova is probably undetectable by an X-ray satellite. However, after a few days, the expanding shell starts to become ionized and the copious X-rays from the inner layers partially penetrate the shell and X-ray observations of novae during this phase show emission lines from the expanding gas (Mukai \& Ishida 2001; Orio et al. 2002, 2003). This is the second phase.

The third is the constant bolometric luminosity phase when the system again becomes observable as a "soft" source. This phase occurs because both observational and theoretical studies of $\mathrm{CNe}$ show that only part of the envelope is ejected during the explosive phase of the outburst. Some fraction remains on the WD and rapidly returns to hydrostatic equilibrium. This material provides sufficient fuel so that the enlarged WD remains hot and luminous for months to years (Krautter et al. 1996). It is predicted that the duration of this phase is an inverse function of the mass of the WD (Krautter et al. 1996) so that a determination of how long a nova is "on" in X-rays can provide an estimate of the WD mass. Moreover, since the burning WD is hot, with nuclear burning ongoing in its surface layers, its characteristics closely resemble those of the SSS.

In this paper we report on the analysis of an observation of nova V4743 Sgr with the High Resolution Camera and the Low Energy Transmission Grating Spectrograph (HRC-S + LETGS) on the CHANDRA X-ray satellite (Ness et al. 2003). Because the structure of the rekindled WD in V4743 Sgr consists of a nuclear burning envelope with a very hot stellar atmosphere on top, we have updated the microphysics and numerics in PHOENIX to analyze nova atmospheres at X-ray wavelengths. We describe these improvements in Sect. 2.2. PHOENIX is well suited to analyze V4743 Sgr, or any SSS, because it has been developed specifically to treat radiation transport in an expanding, optically thick atmosphere at any temperature. We show that we can now treat the formation and time evolution of the $\mathrm{X}$-ray radiation in a $\mathrm{CN}$ in outburst and that it is possible to determine the chemical composition of the $\mathrm{CN}$ ejecta. We describe the X-ray observations of V4743 Sgr in Sect. 3, and end with a Summary and Discussion (Sects. 4 and 5).

\section{Modeling novae in X-rays}

The nova models calculated with the PHOENIX code are 1D spherical, expanding, line blanketed, NLTE ${ }^{1}$ model

\footnotetext{
1 non-local thermodynamic equilibrium.
}

atmospheres. The spherical, co-moving frame radiative transfer equation (SSRT) for expanding media is solved for lines and continua, coupled with the NLTE statistical equilibrium equations (Hauschildt \& Baron 1999). The numerical solution of the radiation transport and multi-level NLTE problems are based on an operator splitting (ALI) method as described in detail by Hauschildt (1992) and Hauschildt \& Baron (1999). Nova atmospheres are far from LTE, therefore, all models have to include NLTE for as many atoms and energy levels as feasible.

The radiative transfer problem is coupled to the energy conservation through the equation of radiative equilibrium (in the co-moving frame) and, therefore, a temperature correction procedure (Hauschildt et al. 2003) is used to iteratively correct the structure so that energy is conserved. Mechanical energy sources, due to the expansion of the nova envelope, as well as convection are negligible for the conditions found in nova atmospheres.

The density profile and the velocity field of the expanding medium are taken from hydrodynamic simulations (Shara 1989; Starrfield 1989). In this case the radiation transport problem effectively decouples from the hydrodynamic equations and the problem is dramatically simplified. Earlier nova models in other wavelength ranges had shown that there are satisfactory results by the use of these simulations (Hauschildt \& Starrfield 1995). Therefore we use the results of the simulations as a first assumption in this stage of our work.

The nova atmosphere is approximated by an expanding but stationary (in time) structure. This implies that the explicit time dependencies in the radiation transfer and hydrodynamics (or in the given density profile and velocity field) can be neglected and that the time evolution of the nova atmosphere can be represented by a sequence of snapshot models. This assumption is valid since the hydrodynamic time scale is much greater than both the radiative time scale and also the excitation and ionization equilibrium time scales (Bath \& Shaviv 1976).

To account for LTE and NLTE line blanketing, the opacities of all important spectral lines have to be included. This also includes the handling of line blends, e.g., due to line broadening. Doppler broadening due to the large scale velocity field is handled through the co-moving frame radiation transport and the Lorentz transformation of the radiation field is used to obtain the observed spectrum in the Euler frame. PHOENIX includes a large number of NLTE lines as well as LTE background lines of species not treated in NLTE (Hauschildt 1993; Hauschildt \& Baron 1995). It solves the line radiative transfer equations without using approximations such as the Sobolev method (see Grinin 2001, and ref. therein). Therefore, all depth-dependent spectral line profiles have to be calculated in the Lagrangian frame. This in turn requires a large number of wavelength points (400000 for the models presented in this paper).

The generation of a model is very time consuming due to the complexity of the problem. The construction of detailed models is possible only through the use of parallel algorithms in the PHOENIX code (Hauschildt et al. 1997; Baron \& Hauschildt 1998; Hauschildt et al. 2001). Therefore, modern parallel computers are used to allow more complex, more sophisticated, and more realistic models. 


\subsection{Physical parameters of a nova model atmosphere}

There are several physical parameters of a nova model atmosphere. First there is the effective temperature $T_{\text {eff. }}$ Together with the reference radius $r_{\text {ref }}$ (radius at $\tau_{\text {std }}=1$, where the optical depth grid is calculated at a standard wavelength, depending on the effective temperature of the model) it defines the bolometric luminosity of the nova via $L_{\mathrm{bol}}=4 \pi \sigma T_{\mathrm{eff}}^{4} r_{\text {ref }}^{2}$. This definition is only used as a convenient parameterization.

There is ample evidence from modeling of the outburst that the nova wind is driven by radiation pressure (Hauschildt \& Starrfield 1994). Therefore, the static Eddington luminosity $L_{\text {edd }}$ cannot be used as an upper limit. Thus the luminosity of the nova has to be considered as a free parameter or taken from hydrodynamical simulations. For the models presented here, we have assumed a bolometric luminosity of $L_{\mathrm{bol}}=50000 L_{\odot}$. This value implies a white dwarf mass close to the Chandrasekhar limit. In general, nova model atmospheres for earlier phases of the outburst have shown very little dependence of the spectrum on the bolometric luminosity (Hauschildt \& Starrfield 1995). This is also true for the models presented in this work and we parameterize our models by the effective temperature.

The density profile of the nova atmosphere can be parameterized as a power law of the form

$\rho(r) \propto r^{-N}$.

The velocity profile follows from the requirement that the mass loss rate $\dot{M}(r, t)$ is constant in space (Bath \& Shaviv 1976), i.e.,

$v(r)=\frac{\dot{M}}{4 \pi r^{2} \rho(r)}$

with two parameters $N$ and $v_{\text {out }}$. For novae, we use a typical value of $N=3 \cdot v_{\text {out }}$ is the outer velocity of the expanding shell, here we use the observed value of $v_{\text {out }}=2500 \mathrm{~km} \mathrm{~s}^{-1}$ determined from the width of the line features of the X-ray spectra shown below. $\rho_{\text {out }}$ determines the outer edge of the envelope and is given by an outer pressure $p_{\text {out }}$ assuming that the ideal gas equation is valid. Reasonable values lie in the range between $p_{\text {out }}=10^{-2}$ and $10^{-1}$ dyn $\mathrm{cm}^{-2}$. Together with $N=3$ and $r^{2}$ in Eq. (2) this leads to a velocity field which is linear in $r$.

\subsection{Additional physics included in PHOENIX for modeling novae in the SSS phase}

In order to model the X-ray spectra of novae with PHOENIX, we had to extend the opacity and line databases into the X-ray spectral range (from $\lambda \approx 1 \AA$ A, equivalent to $E \approx 12.4 \mathrm{keV}$ ). Several new atomic data and physical processes have to be included in order to construct detailed nova atmosphere models with effective temperatures in excess of $10^{5} \mathrm{~K}$. Previously the only database with X-ray data available in PHOENIX was CHIANTI Version 3 (Dere et al. 1997, 2001). For this work, we have extended PHOENIX to also include the CHIANTI Version 4 (CHIANTI4, Young et al. 2003) and the APED
Table 1. Number of spectral lines from the atomic databases. Given are the number of X-ray lines (defined as lines with wavelengths $\lambda \leq$ $100 \AA$ ) and the sum of all lines over the whole spectral range (up to $\lambda \approx 10^{7} \AA$ ). In the APED database different entries for the same transition are possible. In the last row the number of unique lines are specified.

\begin{tabular}{lll}
\hline \hline Database & X-ray lines & All lines \\
\hline CHIANTI Ver. 3 & $\approx 12.650$ & $\approx 45.000$ \\
CHIANTI Ver. 4 & $\approx 22.350$ & $\approx 74.500$ \\
APED (total) & $\approx 326.000$ & $\approx 463.200$ \\
APED (distinct) & $\approx 178.000$ & $\approx 310.400$ \\
\hline
\end{tabular}

(Astrophysical Plasma Emission Database, Version 1.3.1) ${ }^{2}$ databases.

The new databases provide the following extensions compared to previous PHOENIX versions:

- NLTE model atom data for highly ionized species or species with large ionization potential, including;

- many new spectral lines in the X-ray waveband down to $1 \AA$;

- improved data for electron collision rates;

- new data for proton collision rates;

- better data for thermal bremsstrahlung.

All these improvements were used for the models presented in this work.

In Table 1 we give the number of atomic lines found in each of the databases. The APED database provides a good extension to the other databases. Note, that many of the lines in the databases are rather weak but, collectively, are important opacity sources. There are a number of transitions with multiple entries for the same line but different atomic data and it is not clear which entries are better than others. For this work, we simply use the first entry for each unique transition of the line databases and ignore all duplicate data. The effects of using different data where available will be investigated in a later paper.

The values of the electron and proton collision rates for each ion depend significantly on the database. In addition, the proton collision rates are only available in the CHIANTI4 and APED databases. All applicable collision rates are used in the rate equations of PHOENIX for ions whose transitions are treated in NLTE. A detailed investigation of the sensitivity of the results on the collisional data will be presented in a subsequent paper.

Thermal bremsstrahlung is calculated using the data for the ff-gaunt factors available in the databases. Previously, PHOENIX considered only contributions of $\mathrm{H}, \mathrm{Si}$, and $\mathrm{Mg}$ as bremsstrahlung processes (called "old bremsstrahlung" in this paper). In the CHIANTI Version 4 database, the ff-gaunt factors are available for elements up to $Z=30$. These bremsstrahlung processes have been included in this work (called "new bremsstrahlung" in this paper) which is important for highly ionized abundant elements. The nova model atmospheres are

\footnotetext{
${ }^{2}$ http://cxc.harvard. edu/atomdb/
} 
optically thick, therefore, free-free absorption as the inverse process to the bremsstrahlung emission is important and included in the models. Two ranges for the plasma temperature $T$ and the ratio of photon to thermal energy $u$ have to be distinguished. For $T \geq 10^{6} \mathrm{~K}$ and $u=\frac{h v}{k T} \geq 10^{-4}$ (where $v$ is the frequency of the radiation) the relativistic ff-gaunt factors and the fits of Itoh et al. (2000) are used. Outside this range we use the ff-gaunt factors of Sutherland (1998) since they are valid over a large range of temperatures and frequencies. In both references, the ff-gaunt factors are given for elements up to $Z=$ 28. However, it is possible to enhance the fits up to $Z=30$. These extensions are provided in the CHIANTI4 database and used in the model calculations presented here.

\subsection{Computation of hot nova models}

The models computed for this work are the first PHOENIX nova atmosphere models with effective temperatures $T_{\text {eff }} \geq 10^{5} \mathrm{~K}$. Due to the extreme conditions in nova atmospheres, it is better to start the model calculations from scratch rather than using scaled structures from our earlier nova modeling. The first step in this process is to calculate an LTE continuum model without any spectral lines for each temperature, typically starting from a grey temperature structure. In the next step, spectral lines are included in the LTE model to generate a model structure that includes LTE line blanketing. Next, NLTE models are constructed with more and more species treated in NLTE. It is important to treat the transitions of all important ionization stages of one element in NLTE simultaneously, because NLTE effects like over- and under-ionization affect the level populations of adjacent ionization stages. Therefore, large sets of NLTE species are needed in the model atmospheres. However, there are convergence problems if the calculation includes too many "fresh" NLTE species at once. Therefore, we use a multi-stage process to reach the final models with the full set of NLTE species.

Each step needs about 30 iterations and we calculate about 270 iterations in total for a model presented in this publication. The model includes about $7 \times 10^{3} \mathrm{~b}-\mathrm{f}$ and about $100 \times$ $10^{3} \mathrm{~b}-\mathrm{b}$ transitions in NLTE. The atmosphere is divided into 50 layers and distributed along an optical depth grid from $\tau_{\text {std_min }}=10^{-10}$ to $\tau_{\text {std_max }}=10^{2}$.

\section{X-ray observations of nova V4743 Sgr}

Nova V4743 Sgr was first detected on 20 September 2002 by Haseda et al. (2002) at $\mathrm{m}_{V}=5$. An accurate position is $\operatorname{RA}(J 2000)=19^{\mathrm{h}} 01^{\mathrm{m}} 09.38, \operatorname{Dec}(\mathrm{J} 2000)=-22^{\circ} 00^{\prime} 05^{\prime \prime} \cdot 9$ (determined by Tanaka et al. 2002). From the optical light curve the nova was classified as very fast. A velocity of $2400 \mathrm{~km} \mathrm{~s}^{-1}$ was estimated from the full width at half maximum (FWHM) of the $\mathrm{H} \alpha$ emission line, indicating that the expansion velocities are $\sim 1200 \mathrm{~km} \mathrm{~s}^{-1}$. Lyke et al. (2002) determined a distance of $6.3 \mathrm{kpc}$ based on infrared observations. The first X-ray observation of V4743 Sgr was obtained with the ACIS-S instrument on CHANDRA in 2002 November. It was faint at this time and did not appear to be in the SSS phase as yet. We then observed it for $24.7 \mathrm{ks}$ with the HRC-S+LETG on 19 March 2003 (OBSID 3775, Ness et al. 2003; Starrfield et al. 2003).

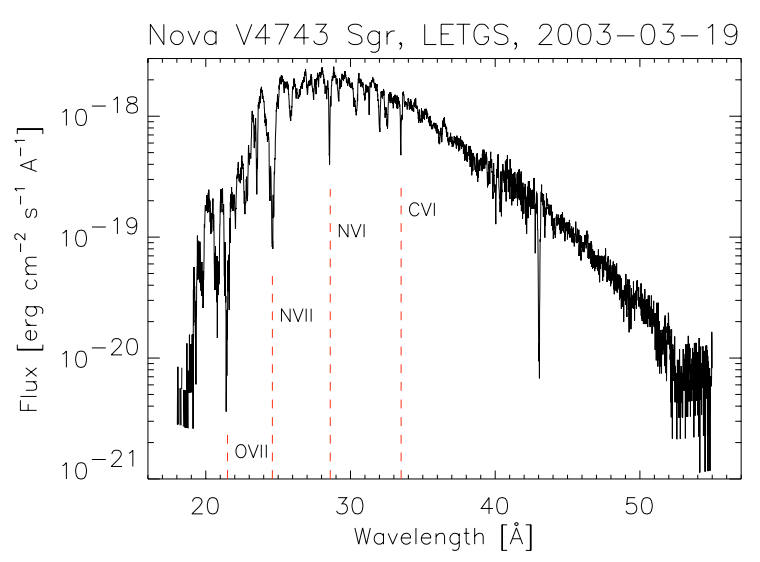

Fig. 1. The observed Chandra LETGS spectrum of nova V4743 Sgr. There are absorption lines and probably weak emission lines in the spectrum. The strongest lines are from the two highest ionisation stages of C, N, and O (Ness et al. 2003).

The light curve for the first grating observation proved extremely interesting with both large amplitude oscillations and, about two-thirds of the way through the observation, a rapid decline to almost no counts per second (Ness et al. 2003). Two further HRC-S+LETGS observations were carried out on 2003 July 18, and 2003 September 26 (11.7 ks: OBSID 3776 and $12.0 \mathrm{ks}$ : OBSID 4435).

The spectrum from the first observation is shown in Fig. 1. The effective areas used to convert from $\mathrm{ct} \mathrm{s}^{-1}$ to flux are determined with the CIAO (Chandra Interactive Analysis of Observations) software package ${ }^{3}$, version 3.0. The spectrum is background subtracted. At wavelengths greater than $\approx 55 \AA$, the spectrum is dominated by second and higher dispersion orders (Ness et al. 2003), and these wavelengths will not be considered in our analysis.

An examination of the spectrum shows that it is not a black-body but resembles a stellar atmosphere with deep absorption features and, possibly, some weak emission lines. This spectrum only slightly resembles that of either CAL 83 (Paerels et al. 2001) or V1494 Aql (Starrfield et al. 2001). The strongest lines are from the two highest ionisation stages of $\mathrm{C}, \mathrm{N}$, and $\mathrm{O}$ (Ness et al. 2003).

An extensive analysis of the observation from March has been carried out by Ness et al. (2003). A remarkable feature in the light curve from that observation is a strong decline of the $\mathrm{X}$-ray emission between 13 and $20 \mathrm{ks}$. Figure 1 of Ness et al. (2003) shows the light curve taken during this observation. It is oscillating at high amplitude with a period of $\sim 22 \mathrm{~min}$. Moreover, the oscillations exhibit spectral variability and a correlated change in hardness ratio, defined by $(h-s) /(h+s)$, with $h$ being the flux in $19 \AA<\lambda<30 \AA$ and $s$ being the flux in $30 \AA<\lambda<50 \AA$. There is a strong period present in the data, with at least two harmonic overtones, which reflect the complex nature of the variations. This is in line with the X-ray oscillations of V1494 Aql which were also multiperiodic and were interpreted as pulsation by Drake et al. (2003). If the oscillations in V4743 Sgr (which exhibited a much larger

\footnotetext{
${ }^{3}$ http://cxc.harvard.edu/ciao/
} 
amplitude than those in V1494 Aql) are interpreted as a rotational modulation then they imply a major asymmetry in the $\mathrm{X}$-ray emitting region.

The spectrum of the last $5.4 \mathrm{ks}$ can be seen in Fig. 4 of Ness et al. (2003). There are strong emission lines but there is no continuum present so we cannot discern if absorption lines are present. In any case, the peak count rate at this time is far lower than in any of the X-ray observations where a strong continuum is present. A possible scenario for the decline at $13 \mathrm{ks}$ involves an eclipse of the X-ray emitting WD. But this does not explain either the long duration of the decline or the softening during the decline. The emission spectrum at minimum is probably produced by the ejected shell and not by a hot stellar photosphere. Therefore, we do not consider it any further in this paper.

\section{Results}

The PHOENIX code package was modified to calculate model atmospheres and synthetic spectra for the late phases of classical novae where most of the energy is emitted in X-rays. Our models have effective temperatures of $T_{\text {eff }}=10^{5}-10^{6} \mathrm{~K}$ and a bolometric luminosity of $L_{\mathrm{bol}}=50000 L_{\odot}$. In the generation of the first test models the "old bremsstrahlung" was used. A typical NLTE model spectrum can be seen in the upper panel of Fig. 2. There are only a few weak absorption lines and the other spectral lines are in emission. Clearly, the observed spectra (Fig. 1) are not well represented by models using the "old bremsstrahlung".

In calculating the other grids of models the "new bremsstrahlung" was used (Fig. 2, lower panel), where there are mostly absorption lines in the spectrum. Considering the interstellar and circumstellar absorption by hydrogen, the shape of the continuum model spectrum is close to what is observed (Fig. 3). The agreement between the continuum of the model and the observation is acceptable, whereas there are differences in the spectral lines.

The best fit to the spectrum of nova V4743 Sgr is found at a temperature of $T_{\text {eff }}=5.8 \times 10^{5} \mathrm{~K}$. To get the correct slope for the pseudo-continuum (continuum formed by numerous overlapping lines), a suitable value of the hydrogen column density has to be used. A value of $n_{h}=4.0 \times 10^{21} \mathrm{~cm}^{-2}$ for the hydrogen column density provides the best fit, in contrast to the value of $n_{h}=1.41 \times 10^{21} \mathrm{~cm}^{-2}$ given by Dickey \& Lockman (1990) for the ISM. This discrepancy can be explained by assuming additional absorption from the circumstellar shell, not included in the value from the literature.

Close inspection of the measured spectrum reveals that some spectral lines are not reproduced well or are missing in the model spectrum. This is because we have used only solar abundances in the model and have not enhanced abundances of, for example the $\mathrm{CNO}$ elements, as is generally observed in novae and predicted by theory. In the best model spectrum with solar abundances, all absorption lines are too weak and there is too much emission around $24 \AA(\approx 517 \mathrm{eV})$. Increasing the abundances should increase the absorption. We will do this in a succeeding publication. There is an oxygen edge around $24 \AA$ A. Increasing the O-abundance should add more opacity
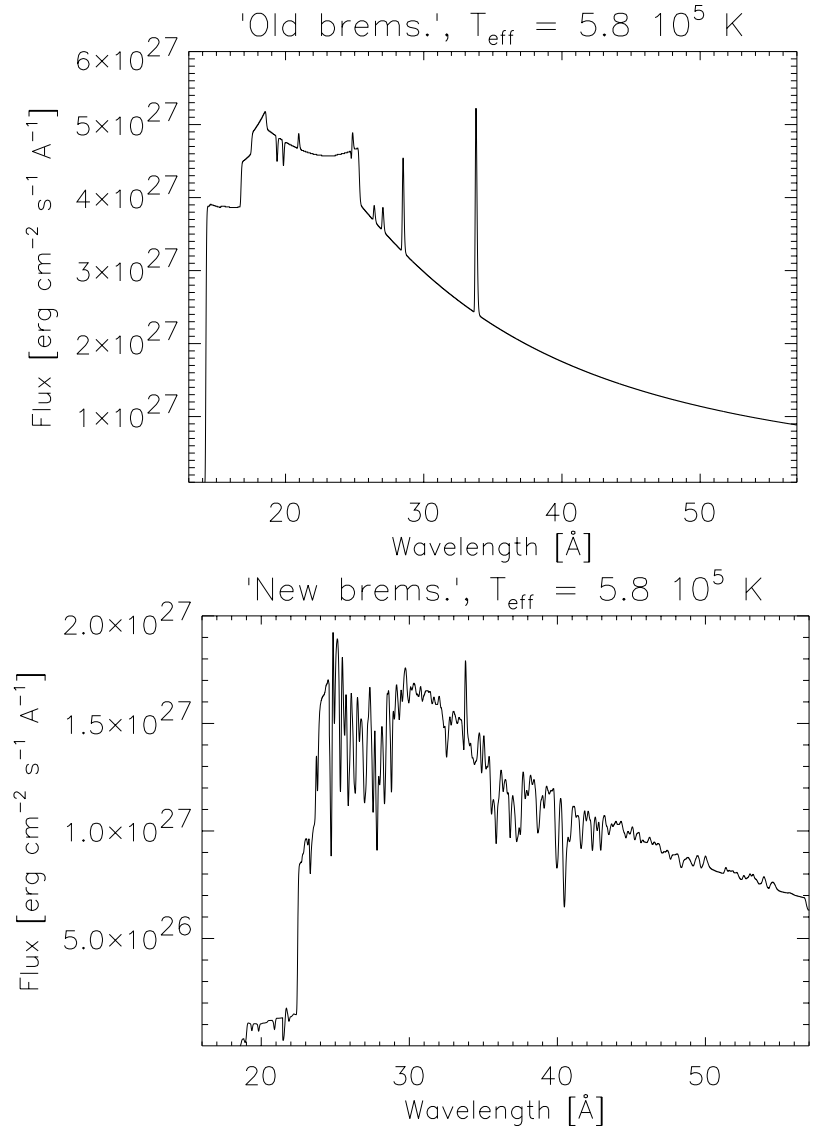

Fig. 2. Upper: model spectrum calculated with the "old bremsstrahlung" continuum. Because these are test models, there are only spectral lines from $\mathrm{H}, \mathrm{He}, \mathrm{C}$, and N. Lower: model spectrum calculated with the "new bremsstrahlung" continuum and spectral lines of $\mathrm{H}, \mathrm{He}, \mathrm{C}, \mathrm{N}, \mathrm{O}$, and $\mathrm{Fe}$. There are mainly absorption lines in the spectrum.

and could provide a better fit. The computation of models with abundances deviating from solar needs much more computational work and has not yet been done. With such models it will be possible to estimate the chemical abundances of the nova ejecta.

The lack of lines in the computed spectrum may also be caused by using only line data from ions of $\mathrm{H}, \mathrm{He}, \mathrm{C}, \mathrm{N}$, and $\mathrm{O}$ and from Fe XXI-XXVI in the model calculations. If the concentration of the partial pressures of an ion is sufficiently large, all available transitions between all available levels of these ions are treated in NLTE. This procedure selects the last three ionization stages of $\mathrm{C}, \mathrm{N}$, and $\mathrm{O}$ to be included in the NLTE calculation and the others of these elements are treated in LTE.

For each ion it can be decided which atomic database will be used. Because many X-ray spectral lines are required, the CHIANTI4 or APED databases are used (Table 1). From a rough comparison, the spectra calculated with each database are only slightly different. The shape of the continuum and the number of strong lines are the same. The quality of the databases has to be determined for an accurate abundance analysis. For the models presented in this paper we have used the APED database. 


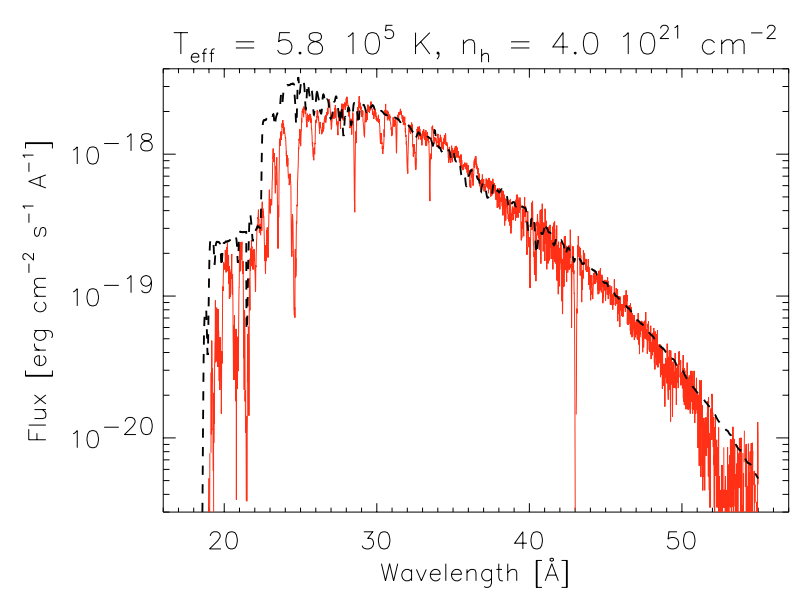

Fig. 3. NLTE Model spectrum (calculated with the "new bremsstrahlung") with solar abundances plotted over the first $15 \mathrm{ks}$ of the March observation. There is a very good agreement. A best-fit is provided with an effective temperature of $T_{\text {eff }}=5.8 \times 10^{5} \mathrm{~K}$ $\left(L_{\text {bol }}=50000 L_{\odot}, v_{\text {out }}=2500 \mathrm{~km} \mathrm{~s}^{-1}, p_{\text {ref }}=10^{-2} \mathrm{dyn} / \mathrm{cm}^{2}, N=3\right)$. The absorption lines and the continuum absorption are too weak in the model. Interstellar absorption was taken into account $\left(n_{h}=4.0 \times\right.$ $10^{21} \mathrm{~cm}^{-2}$ ).

The main results from the models are that the X-ray emission is dominated by thermal bremsstrahlung from the atmosphere surrounding the WD, and that the hard spectral range of $\lambda \lesssim 29 \AA(E \gtrsim 428 \mathrm{eV})$ is dominated by iron and nitrogen absorption. As expected, the departures from LTE are large in the nova ejecta. To examine the strength of NLTE effects, the departure coefficients can be plotted against the standard optical depth $\tau_{\text {std }}$. The departure coefficient $b_{i}$ is the ratio of the occupation numbers in level $i$ calculated in NLTE to calculations in LTE for a specific ion. The stronger the departure from LTE the more different the value of the departure coefficient from 1. In the outer layers of nova atmospheres the departure coefficients can differ up to six dex from 1 in both directions. This can be seen in Fig. 4 for different elements. Spectral lines are formed in regions where the departure coefficients are very different from 1 . Therefore it is very important to treat all atomic transitions in full NLTE.

In addition to strong departures from LTE, there are extreme physical conditions in the nova ejecta. The atmosphere is very extended with a radius of $r \approx 10^{12} \mathrm{~cm}$ at $\tau_{\text {std }}=10^{-10}$ (Fig. 5, upper panel). There is a wide range of electron temperatures (up to several $10^{6} \mathrm{~K}$, Fig. 5, lower panel) and hence several of the highest ionization stages of an element are simultaneously present in the ejecta. For example the partial pressures of iron ions (Fe IX-XXVII) are plotted against the standard optical depth in Fig. 6. A similar effect has already been observed in the IUE analysis of nova spectra (Hauschildt et al. 1992).

\section{Conclusion and outlook}

We have modified the PHOENIX code to calculate model atmospheres and synthetic spectra for the late X-ray phase of classical novae. The agreement between the model and the observed spectrum of nova V4743 Sgr is good in the continuum for an effective temperature of $T_{\text {eff }}=5.8 \times 10^{5} \mathrm{~K}$ if the
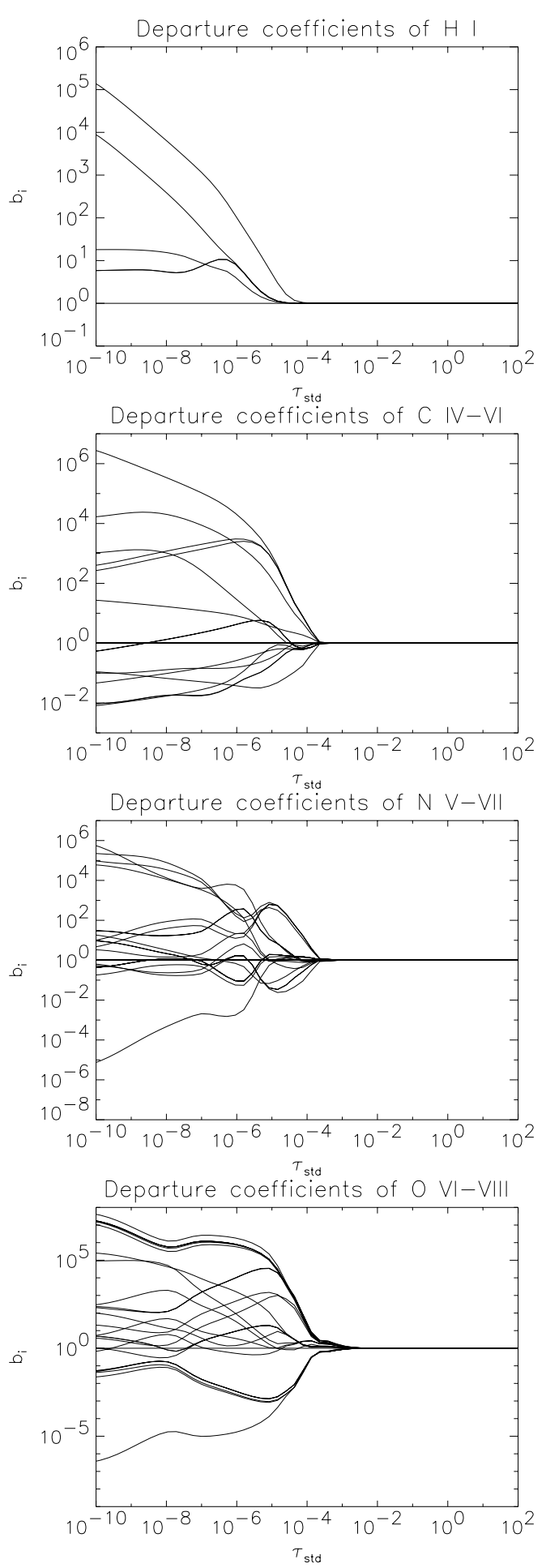

Fig. 4. Departure coefficients $b_{i}$ from the best-fit model for the first 5 levels of the $\mathrm{H}-, \mathrm{C}-, \mathrm{N}-$, and $\mathrm{O}$-ions considered in these models. There are departure coefficients with values of 6 mag larger or smaller than 1. For all elements the departures from LTE are strong. As expected, the departures are strongest for outer layers and there is no departure from LTE at large optical depths $\left(b_{i}=1\right)$.

"new bremsstrahlung" (see Sect. 2.2) is used which is physically more reasonable. So far, we only have computed model atmospheres with solar abundances for computing time reasons and the fit to the spectral lines needs to be improved by models 

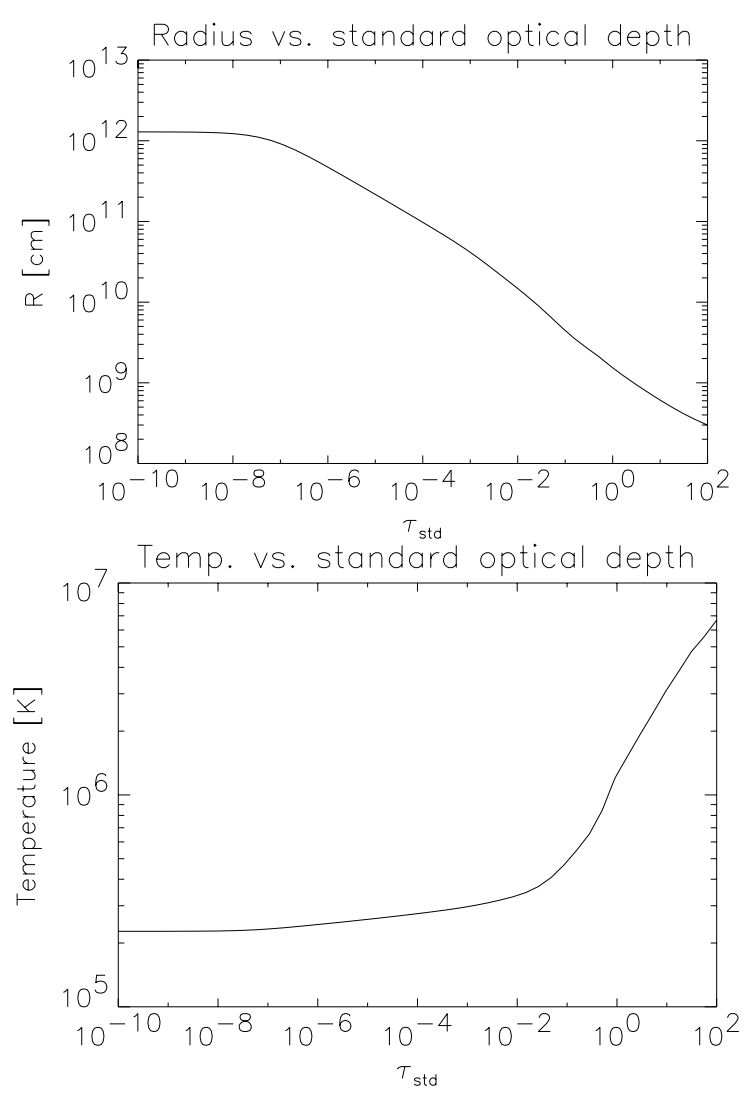

Fig. 5. Upper: radius of the nova atmosphere plotted against the standard optical depth. The nova atmosphere is very extended, with a radius of $r \approx 10^{12} \mathrm{~cm}$ at $\tau_{\text {std }}=10^{-10}$. Lower: temperature distribution in the atmosphere plotted against the standard optical depth. There is a wide variety of temperatures in the atmosphere. In the inner layers the temperature is several $10^{6} \mathrm{~K}$ high.

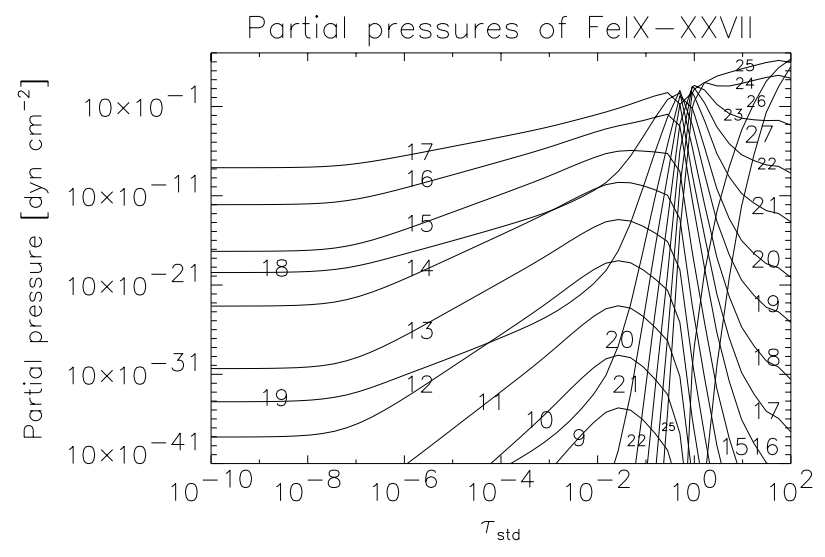

Fig. 6. Partial pressures of FeIX-XXVII. The highest ionization stages of iron exist at the deepest layers of the nova atmosphere. In the outer layers FeXVII is most abundant. The partial pressures of FeI to FeVIII are very small and lie below the range of the $y$-axis. Only ions from FeXV are abundant enough in the line forming region and therefore important.

with non-solar abundances. The hydrogen column density of $n_{h}=4.0 \times 10^{21} \mathrm{~cm}^{-2}$ providing the best fit is high compared to $n_{h}=1.41 \times 10^{21} \mathrm{~cm}^{-2}$ from Dickey \& Lockman (1990), because of additional absorption by the circumstellar shell.
X-ray continuum emission is dominated by thermal bremsstrahlung and its inverse processes, while bound-free emission is much weaker in the nova atmosphere. In the hard spectral range of $\lambda \lesssim 29 \AA(E \gtrsim 428 \mathrm{eV})$ iron and nitrogen absorption is dominant. As expected, nova ejecta show strong departures from LTE. They are very extended and several of the highest ionization stages of all elements are simultaneously present.

In future work additional physical processes absorbing and emitting X-rays will be implemented in the code. For example there are data for dielectronic recombination and autoionization, and there are better data for free-bound coefficients in the APED and CHIANTI4 databases.

Models with non-solar abundances will be calculated to obtain a better fit for the line strengths and the continuum absorption and to analyze the abundances of the nova ejecta. To determine reliable chemical abundances more elements have to be included in the NLTE calculations.

The data quality of the atomic databases as well as differences between the CHIANTI4 and APED database will be checked, e.g. by examining the line profiles. By comparing model and observation, it has to be determined which data of the individual ions reproduce the spectral lines as accurately as possible. This is necessary for an accurate abundance analysis.

The evolution of the X-rays should be examined, by modeling the spectra of other observations of novae with the CHANDRA satellite. It is important to test if it is possible to model the spectrum of novae in the emission line phase, when nuclear burning has switched off and the expanding shell is optically thin, like the spectrum of V382 Vel (Burwitz et al. 2002). For this work more observations with CHANDRA are necessary.

Acknowledgements. Some of the calculations presented here were performed at the Höchstleistungs Rechenzentrum Nord (HLRN) and at the National Energy Research Supercomputer Center (NERSC), supported by the US DOE. We thank all these institutions for a generous allocation of computer time. Part of this work was supported by the DFG (Deutsche Forschungsgemeinschaft), project number HA 3457/2-1. S. Starrfield was partially supported by grants from NASA-CHANDRA, NASA-Theory, and NSF to ASU.

\section{References}

Balman, S., Krautter, J., \& Oegelman, H. 1998, ApJ, 499, 395

Baron, E., \& Hauschildt, P. H. 1998, ApJ, 495, 370

Bath, G. T., \& Shaviv, G. 1976, MNRAS, 175, 305

Burwitz, V., Starrfield, S., Krautter, J., \& Ness, J. U. 2002, in Classical Nova Explosions, AIP Conf. Proc., 637, 377

Dere, K. P., Landi, E., Mason, H. E., Fossi, B. C. M., \& Young, P. R. 1997, A\&AS, 125, 149

Dere, K. P., Landi, E., Young, P. R., \& Zanna, G. D. 2001, ApJS, 134, 331

Dickey, J. M., \& Lockman, F. J. 1990, ARA\&A, 28, 215

Drake, J. J., Wagner, R. M., Starrfield, S., et al. 2003, ApJ, 584, 448

Gehrz, R. D., Truran, J. W., Williams, R. E., \& Starrfield, S. 1998, PASP, 110,3

Grinin, V. P. 2001, Ap, 44, 402

Haseda, K., West, D., Yamaoka, H., \& Masi, G. 2002, in International Astronomical Union Circular, 1 
Hauschildt, P. H. 1992, JQSRT, 47, 433

Hauschildt, P. H. 1993, JQSRT, 50, 301

Hauschildt, P. H., Barman, T., Baron, E., \& Allard, F. 2003, in Stellar Atmosphere Modeling, ASP Conf. Ser., 288, 227

Hauschildt, P. H., \& Baron, E. 1995, JQSRT, 54, 987

Hauschildt, P. H., \& Baron, E. 1999, JCAM, 109, 41

Hauschildt, P. H., Baron, E., \& Allard, F. 1997, ApJ, 483, 390

Hauschildt, P. H., Lowenthal, D., \& Baron, E. 2001, ApJS, 134, 323

Hauschildt, P. H., \& Starrfield, S. 1994, AJ, 108, 1008

Hauschildt, P. H., \& Starrfield, S. 1995, ApJ, 447, 829

Hauschildt, P. H., Wehrse, R., Starrfield, S., \& Shaviv, G. 1992, ApJ, 393, 307

Hillebrandt, W., \& Niemeyer, J. C. 2000, ARA\&A, 38, 191

Itoh, N., Sakamoto, T., Kusano, S., Nozawa, S., \& Kohyama, Y. 2000, ApJS, 128, 125

Kahabka, P., \& van den Heuvel, E. P. J. 1997, ARA\&A, 35, 69

Krautter, J. 2002, in Classical Nova Explosions, AIP Conf. Proc., 637, 345

Krautter, J., Oegelman, H., Starrfield, S., Wichmann, R., \& Pfeffermann, E. 1996, ApJ, 456, 788

Krautter, J., Woodward, C. E., Schuster, M. T., et al. 2002, AJ, 124, 5 Leibundgut, B. 2001, ARA\&A, 39, 67
Lyke, J. E., Kelley, M. S., Gehrz, R. D., \& Woodward, C. E. 2002, Bull. Am. Astron. Soc., 34, 1161

Mukai, K., \& Ishida, M. 2001, ApJ, 551, 1024

Ness, J. U., Starrfield, S., Burwitz, V., et al. 2003, ApJ, 594, L127

Orio, M., Hartmann, W., Still, M., \& Greiner, J. 2003, ApJ, 594, 435

Orio, M., Parmar, A. N., Greiner, J., et al. 2002, MNRAS, 333, L11

Paerels, F., Rasmussen, A. P., Hartmann, H. W., et al. 2001, A\&A, 365, L308

Shara, M. M. 1989, PASP, 101, 5

Starrfield, S. 1989, in Classical Novae, ed. M. F. Bode, \& A. Evans (New York: Wiley), 39

Starrfield, S., Drake, J., Wagner, R. M., et al. 2001, BAAS, 33, 804

Starrfield, S., Ness, J. U., Drake, J. J., \& Orio, M. 2003, in International Astronomical Union Circular, 1

Starrfield, S., Truran, J. W., Wiescher, M. C., \& Sparks, W. M. 1996, in Cosmic Abundances, ASP Conf. Ser., 99, 242

Starrfield, S., Truran, J. W., Wiescher, M. C., \& Sparks, W. M. 1998, MNRAS, 33, 804

Sutherland, R. S. 1998, MNRAS, 300, 321

Tanaka, T., Nishimura, H., Garcia, J., et al. 2002, in International Astronomical Union Circular, 3

Young, P. R., Zanna, G. D., Landi, E., et al. 2003, ApJS, 144, 135 